\title{
Status of the SPIRE Photometer Data Processing Pipelines During the Early Phases of the Herschel Mission
}

C. Darren Dowell ${ }^{1}$, Michael Pohlen ${ }^{2}, 3$, Chris Pearson ${ }^{3}, 4$, Matt Griffin 2 , Tanya Lim ${ }^{3}$, George J. Bendo ${ }^{5}$, Dominique Benielli ${ }^{6}$, James J. Bock ${ }^{1}$, Pierre Chanial ${ }^{7,5}$, Dave L. Clements ${ }^{5}$, Luca Conversi $^{8}$, Marc Ferlet $^{3}$, Trevor Fulton ${ }^{9}$, Rene Gastaud ${ }^{7}$, Jason Glenn ${ }^{10}$, Tim Grundy ${ }^{3}$, Steve Guest $^{3}$, Ken J. King ${ }^{3}$, Sarah J. Leeks ${ }^{3}$, Louis Levenson ${ }^{11}$ Nanyao Lu ${ }^{12}$, Huw Morris ${ }^{3}$, Hien

Nguyen ${ }^{1}$, Brian O'Halloran ${ }^{5}$, Seb Oliver ${ }^{13}$, Pasquale Panuzzo ${ }^{7}$,Andreas Papageorgiou ${ }^{2}$,

Edward Polehampton ${ }^{3,4}$, Dimitra Rigopoulou ${ }^{3}$, Helene Roussel ${ }^{14}$, Nicola Schneider ${ }^{7}$, Bernhard Schulz $^{12}$, Arnold Schwartz ${ }^{12}$, David L. Shupe ${ }^{12}$, Bruce Sibthorpe ${ }^{15}$, Sunil Sidher ${ }^{3}$, Anthony J. Smith $^{13}$, Bruce M. Swinyard ${ }^{3}$, Markos Trichas ${ }^{5}$, Ivan Valtchanov ${ }^{8}$, Adam L. Woodcraft ${ }^{16}$, C. Kevin $\mathrm{Xu}^{12}$, \& Lijun Zhang ${ }^{12}$

${ }^{1}$ Jet Propulsion Laboratory/California Institute of Technology, 4800 Oak Grove Drive, Pasadena, CA 91109, USA;

${ }^{2}$ School of Physics and Astronomy, Cardiff University, The Parade, Cardiff, CF24 3AA, UK;

${ }^{3}$ Rutherford Appleton Laboratory, Chilton, Didcot, Oxfordshire OX11 0QX, UK;

4 Institute for Space Imaging Science, University of Lethbridge, Lethbridge, Alberta, T1K 3M4, Canada

${ }^{5}$ Imperial College London, Blackett Laboratory, Prince Consort Road, London SW7, 2AZ, UK

${ }^{6}$ Laboratoire d'Astrophysique de Marseille, UMR6110 CNRS, 38 rue F. Joliot-Curie, F-13388 Marseille, France

${ }^{7}$ Commissariat à l'Énergie Atomique, Service d'Astrophysique, Saclay, 91191 Gif-sur-Yvette, France

${ }^{8}$ ESA Herschel Science Centre, ESAC, Villanueva de la Cañada, Spain

${ }^{9}$ Blue Sky Spectroscopy Inc., Suite 9-740 4th Avenue South, Lethbridge, Alberta T1J 0N9, Canada

${ }^{10}$ University of Colorado, Dept. of Astrophysical and Planetary Sciences, CASA 389-UCB, Boulder, CO 80309, USA

11 California Institute of Technology, 1200 E. California Blvd., Pasadena, CA 91125, USA

12 NASA Herschel Science Center, IPAC, 770 South Wilson Avenue, Pasadena, CA 91125, USA 13 Astronomy Centre, Dept. of Physics \& Astronomy, University of Sussex, Brighton BN1 9QH, UK

14 Institut d'Astrophysique de Paris, UMR7095, UPMC, CNRS, 98bis Bd. Arago, 75014 Paris, France

${ }^{15}$ UK Astronomy Technology Centre, Royal Observatory, Blackford Hill, Edinburgh EH9 3HJ, UK

${ }^{16}$ SUPA, Institute for Astronomy, University of Edinburgh, Blackford Hill, Edinburgh EH9 $3 \mathrm{HJ}, \mathrm{UK}$

Further author information:

C.D.D.: e-mail cdd@astro.caltech.edu, telephone 1-818-393-5032

M.P.: e-mail Michael.Pohlen@astro.cf.ac.uk, telephone +44 (0)29 208-70168

C.P.: e-mail chris.pearson@stfc.ac.uk

Space Telescopes and Instrumentation 2010: Optical, Infrared, and Millimeter Wave, edited by Jacobus M. Oschmann Jr., Mark C. Clampin, Howard A. MacEwen, Proc. of SPIE Vol. 7731,773136 - ( 2010 SPIE - CCC code: 0277-786X/10/\$18 - doi: 10.1117/12.858035 


\begin{abstract}
We describe the current state of the ground segment of Herschel-SPIRE photometer data processing, approximately one year into the mission. The SPIRE photometer operates in two modes: scan mapping and chopped point source photometry. For each mode, the basic analysis pipeline - which follows in reverse the effects from the incidence of light on the telescope to the storage of samples from the detector electronics - is essentially the same as described pre-launch. However, the calibration parameters and detailed numerical algorithms have advanced due to the availability of commissioning and early science observations, resulting in reliable pipelines which produce accurate and sensitive photometry and maps at 250,350, and $500 \mu \mathrm{m}$ with minimal residual artifacts. We discuss some detailed aspects of the pipelines on the topics of: detection of cosmic ray glitches, linearization of detector response, correction for focal plane temperature drift, subtraction of detector baselines (offsets), absolute calibration, and basic map making. Several of these topics are still under study with the promise of future enhancements to the pipelines.
\end{abstract}

Keywords: Herschel Space Observatory, far infrared, imaging, bolometer

\title{
1. INTRODUCTION
}

The Herschel Space Observatory was launched on 2009 May 14, achieved first light on the way to L2 orbit over the period 2009 June 22-24, and is expected to have cryogenic operation through early $2013 .{ }^{1}$ Herschel is a $3.5 \mathrm{~m}$ telescope passively cooled to $\sim 85 \mathrm{~K}$ and has three instruments cooled to liquid helium temperatures and sensitive to light over the range $\lambda=60$ to $670 \mu \mathrm{m}$. The SPIRE instrument consists of a three-band imaging photometer and a two-band imaging Fourier-transform spectrometer. ${ }^{2}$ In this paper, we describe the standard data analysis pipelines for the SPIRE photometer.

For continuum imaging, Herschel is a high background situation; at $350 \mu \mathrm{m}$, for example, the emission from the telescope is $\sim 30,000$ times brighter than the spatial 'confusion noise' from the multitude of distant galaxies, blended by diffraction. The large background dictates that the continuum measurements must be comparative in nature. Some SPIRE observations use the classic technique of chopping - modulation of the signal by rapid switching of a mirror between two positions so that the detector alternately sees source plus background and background only. However, this observing mode is inefficient for mapping, so its use is restricted to point source photometry. For covering larger areas, and for denser sampling of the sky plane, scan mapping is used instead. During scan mapping, all instrument and telescope mirrors are held fixed with respect to each other, but the entire observatory is scanned across the sky. Scanning modulates the signal and, especially in the case of crossing scans, allows the reconstruction of accurate maps of relative flux density, but does not permit measurements of absolute surface brightness. Since the confusion limit can be reached rather quickly with the SPIRE photometer, many programs make scan maps much larger than the size of the focal plane detector arrays.

The SPIRE photometer has three detector arrays - 139 feedhorn-coupled bolometers at $250 \mu \mathrm{m}$ ("PSW"), 88 at $350 \mu \mathrm{m}$ (PMW), and 43 at $500 \mu \mathrm{m}$ (PLW) - which observe simultaneously but are analyzed independently in the standard pipelines. Bolometers are well suited for making the comparative measurements described in the previous paragraph. As described elsewhere, ${ }^{3}$ the SPIRE bolometers are read out in a way which gives the bolometer temperature (or voltage) as a function of time over the frequency range 0 to $\sim 5 \mathrm{~Hz}$. Changes in radiation incident on the detector are registered as small changes in temperature (or voltage) in the bolometer, giving an 'AC' signal superposed on a large baseline (DC offset) which is a function of background radation power, detector operating temperature, and individual detector properties. The baseline must be removed in the data analysis. The SPIRE bolometers have very low ' $1 / f$ ' noise ${ }^{2}$ and can measure with background-photon-limited sensitivity signals modulated with frequencies from $\sim 5 \mathrm{~Hz}$ to below $0.01 \mathrm{~Hz}$. In addition to the bolometers, the detector arrays contain a handful of diagnostic channels: dark detectors (bolometers not exposed to incoming light), thermistors (well heat sunk to the silicon detector wafers), and fixed resistors.

The authors of this paper overlap significantly with the SPIRE Instrument Control Centre (ICC), which has the responsibility to deliver scientifically useful SPIRE products to the outside observer community. Since most photometer observations are done in scan map mode, that is the focus of this paper. Section 2 describes the general characteristics of the scan map pipeline, and Section 3 gives details about the modules in that pipeline. Section 4 summarizes the pipeline for the only released SPIRE photometer observing mode that uses 
chopping. Sections 5 and 6 discuss aspects in common to both pipelines. Although the large number of SPIRE scientific publications to date demonstrates the quality of the products emerging from the standard pipelines, improvements can be made. Some future possibilities are discussed in Section 7.

\section{SPIRE PHOTOMETER SCAN-MAP PIPELINE}

The SPIRE data analysis pipelines are based on the sequence of effects on the radiometric signal as it propagates through the telescope, instrument optics, detector, and electronics. The modules in each pipeline attempt to 'undo' each effect in reverse order for the purpose of yielding the true flux densities of astronomical sources. Although in principle it is possible to construct a pipeline which solves for the thermal balance of the bolometer for each time sample, we decided early on to implement as top priority an empirical pipeline. ${ }^{3}$ Calibration within the pipeline is based on in-flight measurements approximating, to first and second order, departures from equilibrium for a bolometer at nominal operating temperature observing nominal dark sky.

The SPIRE electronics and scan map pipeline, as originally conceived, have been described previously. ${ }^{3}$ In this paper, we describe the updates to the pipeline as of one year after launch, illustrate some of the pipeline modules using flight data, describe in detail options and decisions about module operation and calibration, and finally list elements of the pipeline which are in continuing development. We focus on the theoretical underpinnings of the pipeline; for the practical aspects, the reader is referred to the on-line documentation.*

The SPIRE pipeline is operated within the Herschel Interactive Processing Environment ${ }^{4}$ (HIPE). As of this writing, the current version of HIPE is version 4, and our pipeline description applies to that version.

The scan map pipeline flowchart is shown in Figure 1. Changes since the prior publication ${ }^{3}$ are as follows: i) A module has been added to buffer the detector timelines to prevent edge effects, as well as a module to later trim those buffers; ii) Deglitching and electrical crosstalk removal have been reversed, since strong glitches are observed to produce crosstalk; and iii) A module has been added to remove residual detector baselines.

\section{SCAN-MAP PIPELINE DETAILS}

Our presentation of the pipeline modules here is in the order of the data analysis, which is in reverse order of the radiation collection, detection, analog processing, digitization, and storage. In several places, we refer to a timeline (also known as a timestream), which is a time-ordered array of a quantity such as a bolometer signal.

Most SPIRE observations are obtained with nominal detector settings, which have a flux density upper limit ${ }^{2}$ of $200 \mathrm{Jy}$. Objects an order of magnitude brighter can be observed with bright-source settings. Data processing is very similar, with the only differences being some of the calibration files and the thermometers used for temperature drift correction (Section 3.7).

\subsection{Organize Timelines, Convert to Voltage Units}

Each SPIRE scan map Astronomical Observing Template (AOT; Table 1) consists of two or more scanlines, each with nominally constant velocity; the slews between scanlines (turnarounds); and a PCAL (internal far-infrared emission source) calibration. Only the scanlines themselves are included in the standard output data product, and each scanline is processed independently and sequentially to minimize the amount of data which must be kept in memory.

Raw telemetry forms the initial 'Level 0' product of the pipeline. At the 'Level 0.5' stage, detector and thermometer signals have been converted from raw units to voltages and temperatures, and samples have been organized into discrete scanline and turnaround data according to their Building Block ID (BBID). The heart of the scan-map pipeline is a loop over scanline BBID's, accomplishing the analysis steps described in Sections 3.2 through 3.11 .

\footnotetext{
*Herschel Observers' Manual: http://herschel.esac.esa.int/Docs/Herschel/html/observatory.html SPIRE Observers' Manual: http://herschel.esac.esa.int/Docs/SPIRE/html/spire_om.html

HIPE: http://herschel.esac.esa.int/HIPE_download.shtml

HSPOT help: http://herschel.esac.esa.int/Docs/HSPOT/html/hspot-help.html
} 


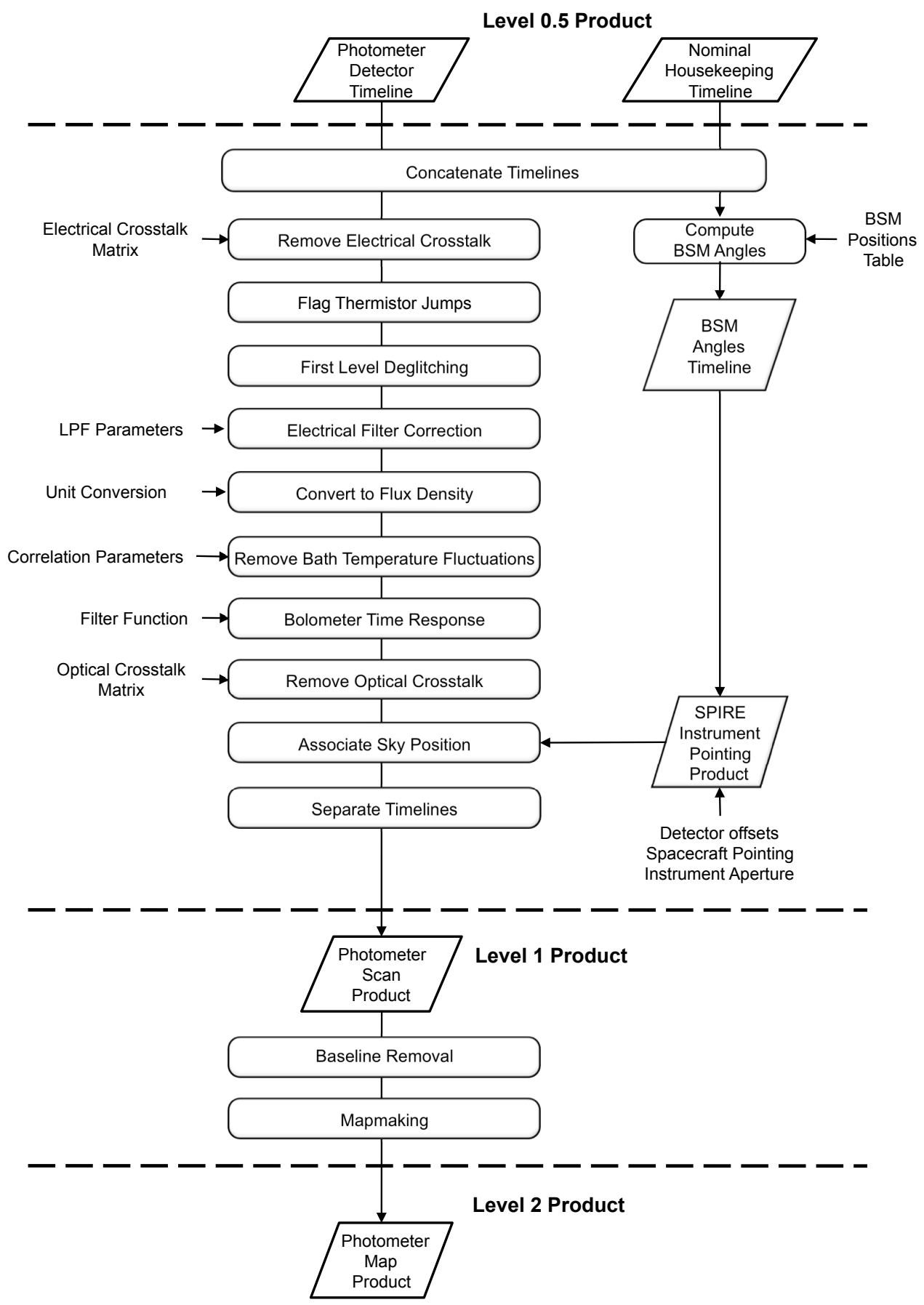

Figure 1. The pipeline flowchart for the SPIRE Scan Map Mode showing the processing steps from the Level 0.5 data through to the final Level 1 and Level 2 data products. Processing tasks are shown as rounded rectangles, data products as parallelograms, and calibration files as plain text. 
Table 1. Released SPIRE Photometer Scan Map Modes

\begin{tabular}{|c|c|c|c|}
\hline AOT Name & $\begin{array}{c}\text { Scan Rate } \\
{\left[{ }^{\prime \prime} / \mathrm{sec}\right]}\end{array}$ & $\begin{array}{c}\text { Sample Rate } \\
{[\mathrm{Hz}]} \\
\end{array}$ & Description \\
\hline "Large Map (POF5) & 30 or 60 & $\overline{18.6}$ & $\begin{array}{l}\text { minimum } 2 \text { scans; single direction (nominal or orthog.), } \\
\text { or cross-linked; maximum field } \sim 3.8^{\circ} \text { squared; } \\
\text { multiple repetitions allowed }\end{array}$ \\
\hline Small Map (POF10) & 30 & 18.6 & $\begin{array}{l}\text { fixed, cross-linked } 1 \times 1 \text { scan covering a field of } 4^{\prime} \text { radius; } \\
\text { multiple repetitions allowed }\end{array}$ \\
\hline $\begin{array}{l}\text { SPIRE PACS Parallel } \\
\text { (PMODE) }\end{array}$ & 20 or 60 & 10.0 & $\begin{array}{l}\text { single direction (nominal or orthog.); } \\
\text { minimum field } 30^{\prime} \times 30^{\prime}\end{array}$ \\
\hline
\end{tabular}

\subsection{Extend Detector Timelines}

Processing of timelines cut to include only the nominal on-source data (as implied in Section 3.1) has some negative consequences. Two of the pipeline modules use Fourier techniques, which cause ringing at the edges of the timelines if the first and last data points are not the same. The temperature drift correction may have similar 'edge effects'. Also, deglitching at the edge of timelines is problematic. To circumvent these problems, each scanline is extended to include the neighboring turnaround building blocks.

\subsection{Correction for Electrical Crosstalk}

The module to correct for electrical crosstalk is the same as described pre-flight. ${ }^{3}$ Since the crosstalk is small $(<0.1 \%)$, a 'dummy' (identity) matrix is used for the calibration file at the present time. However, constructing a suitable crosstalk matrix is a medium priority within the SPIRE ICC.

We are considering two methods to derive the crosstalk matrix. The first is to use cosmic ray impacts on the bolometer webs as a source of isolated signal. A preliminary analysis of flight data indicates that crosstalk matrix elements may be derived by accumulating many well-detected glitches in one bolometer and examining the average response in each other detector channel. The typical crosstalk measured using this method is of order $0.01 \%-0.1 \%$

The second method is to attribute the crosstalk to the nonzero impedance of the bias supply and to calculate the crosstalk accordingly. All of the bolometers and diagnostic devices in a detector array share the same bias supply, and the current from one device pulls down the bias voltage for itself and the other ones. The bolometer voltage can be restored as if the bias supply had zero impedance (i.e., 'de-crosstalked') as follows:

$$
V_{j}^{\prime}=V_{j}+\frac{Z_{j}}{R_{L j}+Z_{j}} \frac{V_{b} \sum_{i} \frac{1}{R_{L i}}-\sum_{i} \frac{V_{i}}{R_{L i}}}{\frac{1}{Z_{b}}+\sum_{i} \frac{1}{R_{L i}}},
$$

where $V_{j}$ and $V_{j}^{\prime}$ are the voltage of bolometer $j$, before and after correction; $Z_{j}$ is the bolometer dynamic impedance, estimated from $I-V$ curves; $R_{L j}$ is the bolometer load resistance; $V_{b}$ is the bias voltage; and $Z_{b}$ is the bias impedance. The observations from the cosmic ray impacts and predictions from this 'bias droop' model will be compared as a step toward providing crosstalk correction of SPIRE data.

\subsection{Deglitching (First Level)}

For a given SPIRE bolometer in flight, approximately $0.3 \%$ of samples are affected by obvious cosmic ray glitches. About half of the glitches are isolated to single bolometers ('web hits'), and the remainder are concurrent within all bolometers and thermistor channels in a detector array ('frame hits'). The concurrent glitches were not anticipated in the pre-flight pipeline development, but have been seen in past space missions with thermal detectors. ${ }^{5}$

Ordinary web-hit and frame-hit glitches can be removed from the data in a straightforward manner, since the bolometers recover quickly (Figure 2). The SPIRE scan-map pipeline currently provides two alternatives for 


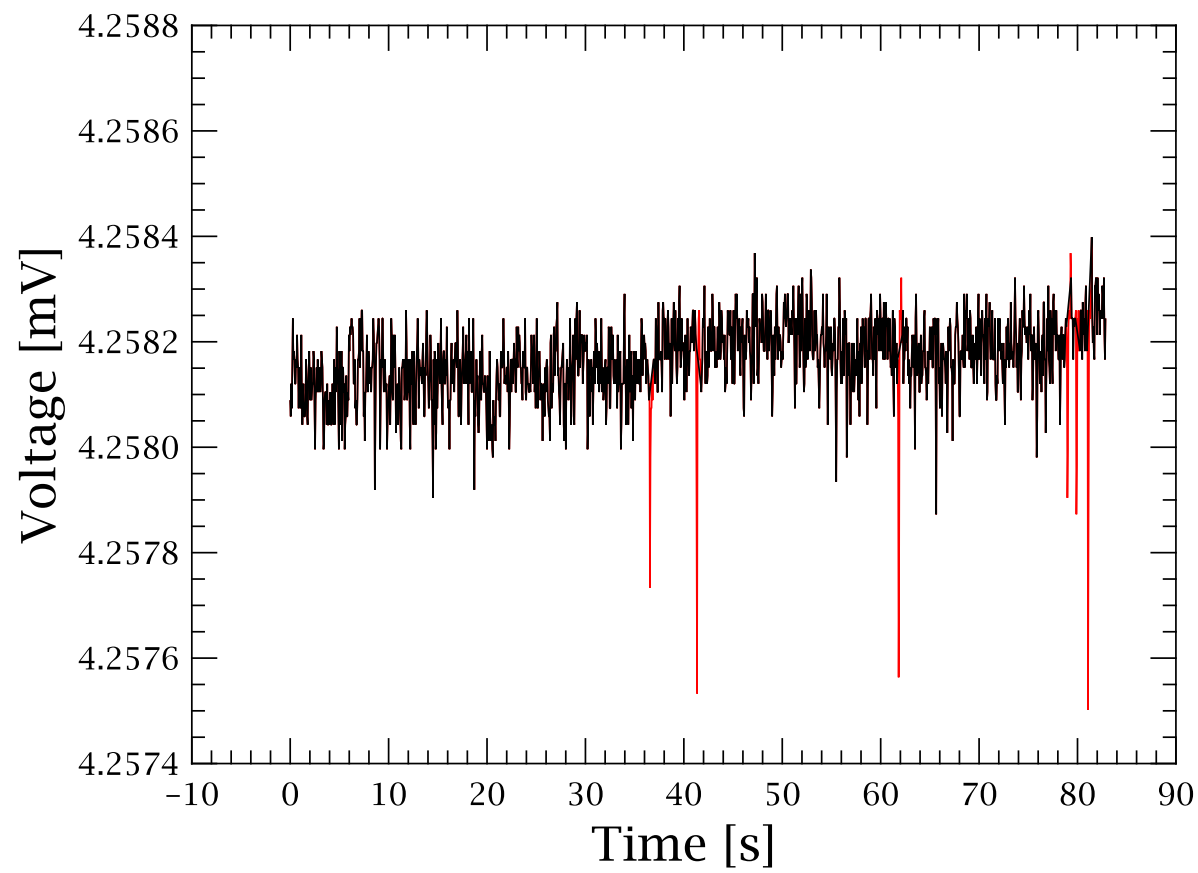

Before wavelet deglitcher

After wavelet deglitcher

Figure 2. Example SPIRE bolometer timeline in flight, with valid samples shown in black and cosmic ray glitches shown in red. These glitches are thermal events, always producing an impulsive temperature rise and hence negative voltage deflection.

first-level deglitching of the detector timelines. Both operate without knowledge of the underlying sky across which the detectors scan, and both use the different time and frequency characteristics of sources and glitches to distinguish them: glitches are rapid and produce signal power over the full signal bandwidth, while sources are confined to low frequencies by diffraction. The default deglitcher, based on wavelet transforms, ${ }^{6}$ has recently been re-optimized for HIPE version 4. At the present time, it appears to offer better all-around performance. The alternative sigma-kappa deglitcher first high-pass filters the timelines with simple operations in the time domain, then performs outlier detection. Once glitches have been identified and masked for exclusion from the maps, both algorithms currently perform linear interpolation over the glitches to provide continuous timelines for intermediate data processing.

The most challenging situations for deglitching are SPIRE-PACS Parallel mode, when the lower sample rate reduces the available bandwidth for distinguishing glitches and sources, and scan timelines containing bright sources. Although compromise deglitching parameters have been chosen for the pipeline to handle typical situations, some customization may be necessary, depending on science goals of a particular observation. Further ICC study on this topic may result in standard 'recipes' for deglitching.

An additional pipeline module to remove concurrent web-hit glitches is offered in HIPE version 4 on an experimental basis. It operates on average timelines over a detector array rather than individual ones, and its approach is otherwise similar to the sigma-kappa deglitcher.

There is a third kind of glitch artifact occasionally affecting a bolometer or diagnostic channel on the SPIRE arrays, which has been tentatively associated with a cosmic ray impact on the amplifier electronics. The signature of the glitch is a sudden jump to a lower voltage, followed by a slow $(\sim 1000 \mathrm{sec})$ rise to the original voltage. This type of glitch is most troublesome in the thermistor channels since they are used to correct for base temperature drift (Section 3.7). Without correction, the worst of these thermistor jumps produce scan lines in the maps with obvious streaks and steps. For Science Demonstration Phase, many investigators coped by eliminating affected 
scan lines from the maps. However, in HIPE version 4, we expect a thermistor jump detector module to be available. It operates by flagging step-like glitches so that the affected portion of the thermistor signal can be disregarded in the temperature drift correction step later on.

The scan-map pipeline currently has only a first level of deglitching. Plans for a second level of deglitching are discussed in Section 7.

\subsection{Correction for Electronic Filtering}

For accuracy, we have adopted a Fourier approach to correct for the on-board analog electronics filtering. The correction consists of using a Fourier transform to go from time domain to frequency domain, dividing by the following transfer function (a 4-pole Bessel filter), then inverse Fourier transforming back to the time domain:

$H(\omega)=\left[\frac{1}{1+i \omega\left(42.6 \times 10^{-3} \mathrm{~s}\right)+(i \omega)^{2}\left(5 \times 10^{-4} \mathrm{~s}^{2}\right)}\right]\left[\frac{1}{1+i \omega\left(25 \times 10^{-3} \mathrm{~s}\right)+(i \omega)^{2}\left(4 \times 10^{-4} \mathrm{~s}^{2}\right)}\right]\left[\frac{1}{1+i \omega\left(10^{-3} \mathrm{~s}\right)}\right]$

The buffer of time samples before and after the nominal scan (see Section 3.2) prevents edge ringing from affecting the maps. Following this step, the noise spectra of the bolometer signals are nearly white up to the highest frequencies.

\subsection{Convert to Flux Density}

The conversion from voltage to flux density (Jy) involves two key components which are incorporated into a single calibration file and pipeline module. ${ }^{7}$ First, the nonlinear response of the bolometer must be corrected. Second, an absolute calibrator must be used to scale linearized voltage to Jy.

The empirical function used to fit the bolometer nonlinear response, developed on ground-based data ${ }^{3}$ and now validated with flight observations (Figure 3), is given by:

$$
\frac{d S}{d V}=K_{1}+\frac{K_{2}}{V-K_{3}}
$$

where $K_{1}, K_{2}$, and $K_{3}$ are fit constants for a bolometer, $V$ is the bolometer voltage, and $S$ is a linearized quantity proportional to flux density. $\frac{d S}{d V}$ is measured with the PCAL source, so this initial $S$ can be thought of as having PCAL intensity units.

During the PCAL calibration step, the telescope was pointed at parts of the sky covering a wide range in surface brightness (in particular, the continuously-viewable Dark Sky field, and the Galactic center). At each pointing, the PCAL source was flashed on and off several times with fixed amplitude. The response of each bolometer was measured and compiled. Finally, the $K_{i}$ parameters were determined from a noise-weighted leastsquares fit (Figure 3). For nominal detector settings, the nonlinearity correction is estimated to be accurate to better than $1 \%$.

The absolute calibration will be based on Neptune. ${ }^{8,9}$ SPIRE observations of this planet are made routinely for the purpose of adjusting the $K_{1}$ 's and $K_{2}$ 's to produce the adopted flux densities from the pipeline.

Since $\frac{d S}{d V}$ is the differential relationship of flux density to voltage, the pipeline module uses instead the integrated form ${ }^{3}$ of Equation 3. At the time of the release of HIPE 4.0, the nonlinearity correction is still based on pre-flight bolometer modeling (while the absolute calibration is based on primary and secondary standards). This is a large contributor to the overall absolute flux calibration uncertainty of $15 \%$ at present. ${ }^{7}$ Implementation later this summer of a new flux conversion calibration file based solely on in-flight measurements is an important step toward pushing the absolute calibration uncertainty down toward the $\sim 5 \%$ uncertainty of the primary standards. 


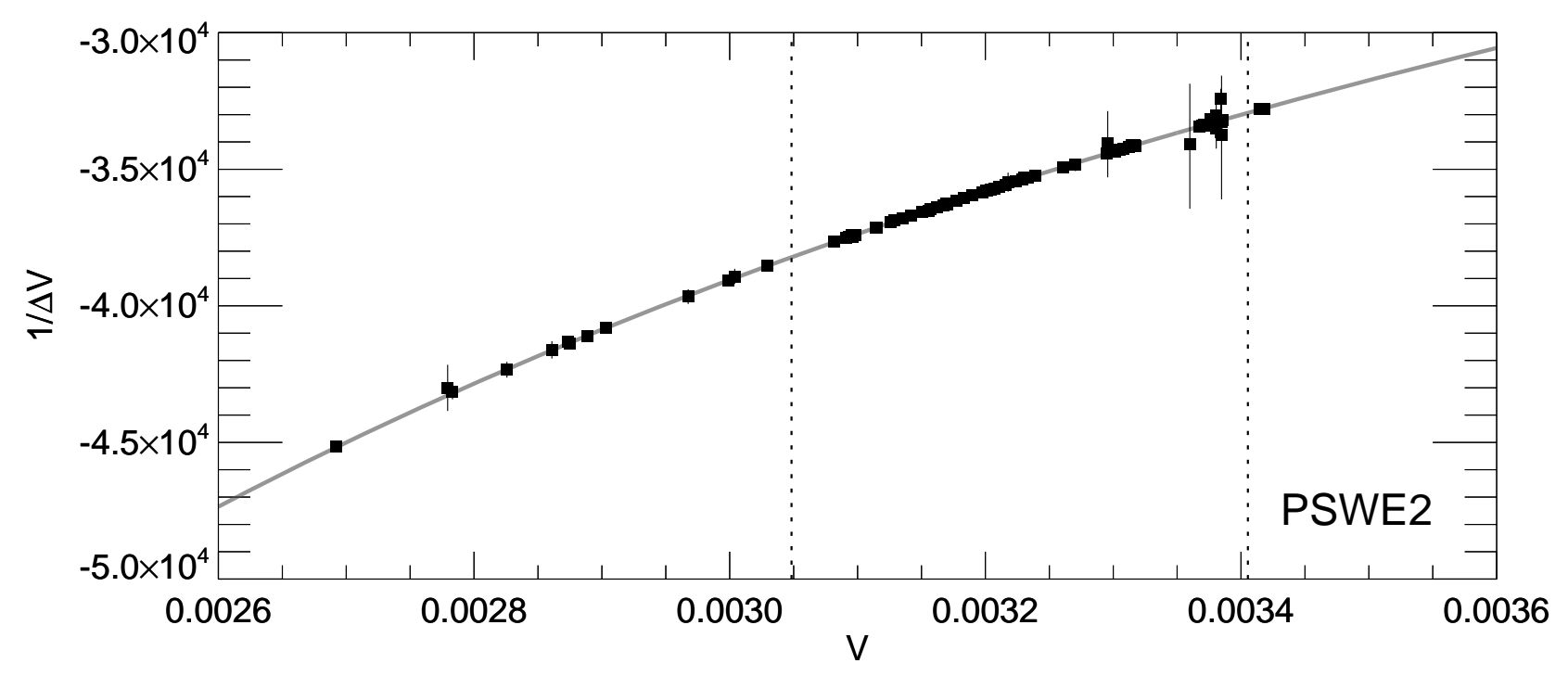

Figure 3. Inverse bolometer response vs. bolometer voltage from a nonlinearity calibration of SPIRE bolometer PSWE2. The units are $\mathrm{V}^{-1}$ and $\mathrm{V}$, respectively. The vertical axis shows the reciprocal of the bolometer response to flashes of the PCAL emission source (response being intrinsically negative for semiconducting bolometers); the reciprocal is used since it is the function $\frac{d S}{d V}$ which is to be fit. Each point is from a different telescope pointing - low surface brightness targets at right, and high surface brightness targets at left. The solid curve shows the best fit with Equation 3 . The right dashed vertical line shows the bolometer voltage for the Dark Sky field, and the left line shows the bolometer voltage for Neptune.

\subsection{Correction for Drift in Detector Base Temperature}

SPIRE is operated without temperature control of the detectors, and the bolometers experience $\sim 2 \mathrm{mK}$ drifts originating at the ${ }^{3} \mathrm{He}$ refrigerator over the course of its two day hold time. It can be shown from bolometer theory ${ }^{\dagger}$ that a base temperature fluctuation can be associated with an equivalent change in power incident on the bolometer, so it is appropriate to apply temperature drift correction after the conversion from voltage to a power unit (Section 3.6). The effect of the temperature drift on the timelines is quite large $-\sim 10 \mathrm{Jy} / \mathrm{mK}-$ so correction is essential. A passive filter with time constant $\sim 400 \mathrm{~s}$ (formed by the heat capacity of the detector arrays and their thermal link with the fridge) attenuates the rapid temperature fluctuations from the fridge, so it is primarily frequencies $<0.1 \mathrm{~Hz}$ which need to be corrected.

The standard pipeline removes from each bolometer a flux density which is proportional to the voltage excursion of the functional thermistors on the same array. For purposes of low-pass filtering, the thermistor signal is binned and fit with a cubic spline function. ${ }^{10}$ The proportionality constants are determined in a separate, dedicated calibration measurement. Figure 4 shows an example application of this pipeline module to flight data.

\subsection{Correction for Bolometer Time Filtering}

Except for a handful of bolometers which are masked by the pipeline to be excluded from the maps, the radiation response of the bolometer itself appears to be consistent with a single-pole filter. Therefore, the timestreams are

\footnotetext{
${ }^{\dagger}$ Bolometer equilibrium is governed by: $\frac{V^{2}}{R}+Q=\int_{T_{0}}^{T} G\left(T^{\prime}\right) d T^{\prime}$, where $V$ is the bolometer equilibrium voltage, $R(T)$ is the bolometer resistance, $Q$ is the radiation power on the bolometer, $T$ is the bolometer temperature, $T_{0}$ is the base temperature, and $G(T)$ is the bolometer thermal conductance. The SPIRE bolometers are biased with a voltage divider such that $V=\frac{R V_{b}}{R+R_{L}}$, where $V_{b}$ is the fixed bias voltage and $R_{L}$ is the fixed load resistance. It can be shown that $\frac{d V}{d T_{0}}$ (fixed $Q)=G\left(T_{0}\right) \frac{d V}{d Q}$ (fixed $T_{0}$ ), i.e. base temperature fluctuations are equivalent to radiation power fluctuations except for a fixed proportionality constant $G\left(T_{0}\right)$.
} 


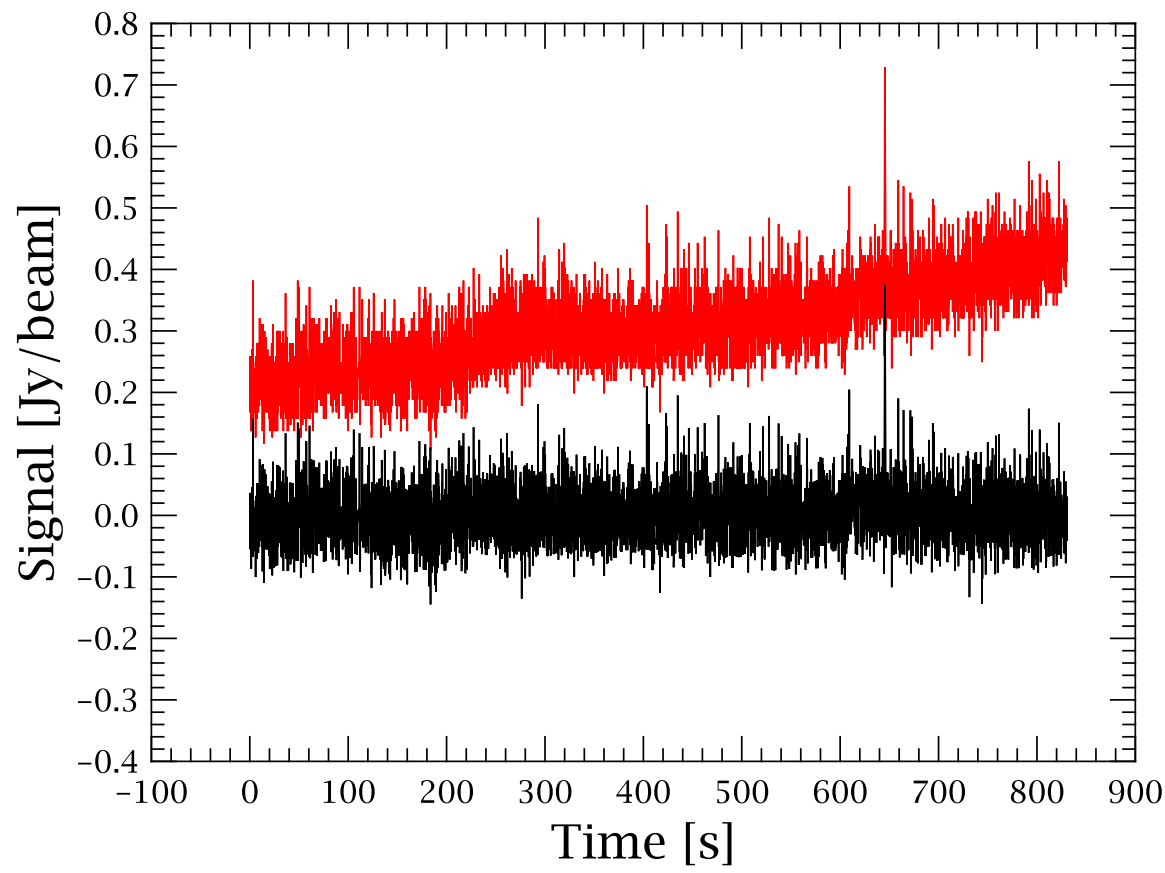

Before T.Drift Correction $\longrightarrow$ After T.Drift Correction

Figure 4. Example SPIRE bolometer timelines before and after correction for temperature drift. Before correction, the timeline has a large $0.25 \mathrm{Jy}$ slope which is absent in the corrected timeline.

corrected with a simple transfer function:

$$
H(\omega)=\frac{1}{1+i \omega \tau_{b o l}},
$$

using Fourier methods as in Section 3.5. The $\tau_{b o l}$ were measured pre-flight with analog electronics with higher bandwidth than the flight unit. At the highest signal frequencies, the noise is raised above the white noise floor due to the declining bolometer response and presence of Johnson and amplifier noise.

\subsection{Correction for Optical Crosstalk}

This module currently uses also a 'dummy' calibration file which leaves the input data unchanged. This will continue to be the case for the foreseeable future, since the known slight imperfections in the optics are best corrected with an approach that uses knowledge of the full image plane rather than just the contemporaneous detector samples. ${ }^{3}$

\subsection{Merge Detector Samples with Telescope Pointing}

At this stage in the pipeline, the calibrated detector signal timelines are merged with the telecope pointing timeline to create Pointed Photometer Timelines. This is a three step process. Starting from the housekeeping data product containing the measured encoder positions of the Beam Steering Mirror (BSM), a BSM angle timeline is created using the associated calibration product of encoder position with respect to the offset from the BSM home position. Since during a scan map the BSM is not moving, this is de facto a constant timeline. Second, a pipeline module calculates the position of every bolometer on the sky. This module has four inputs: a) the timeline of the position of the telecope boresight (as provided in the spacecraft telemetry), b) the positional offset of the SPIRE boresight (coincident with bolometer PSWE8) from the telescope boresight, c) the relative offset of all the SPIRE bolometers from the SPIRE boresight, and d) the above calculated BSM angle timeline. The output is a product representing the position of the bolometers on the sky at each timestep of the telescope 

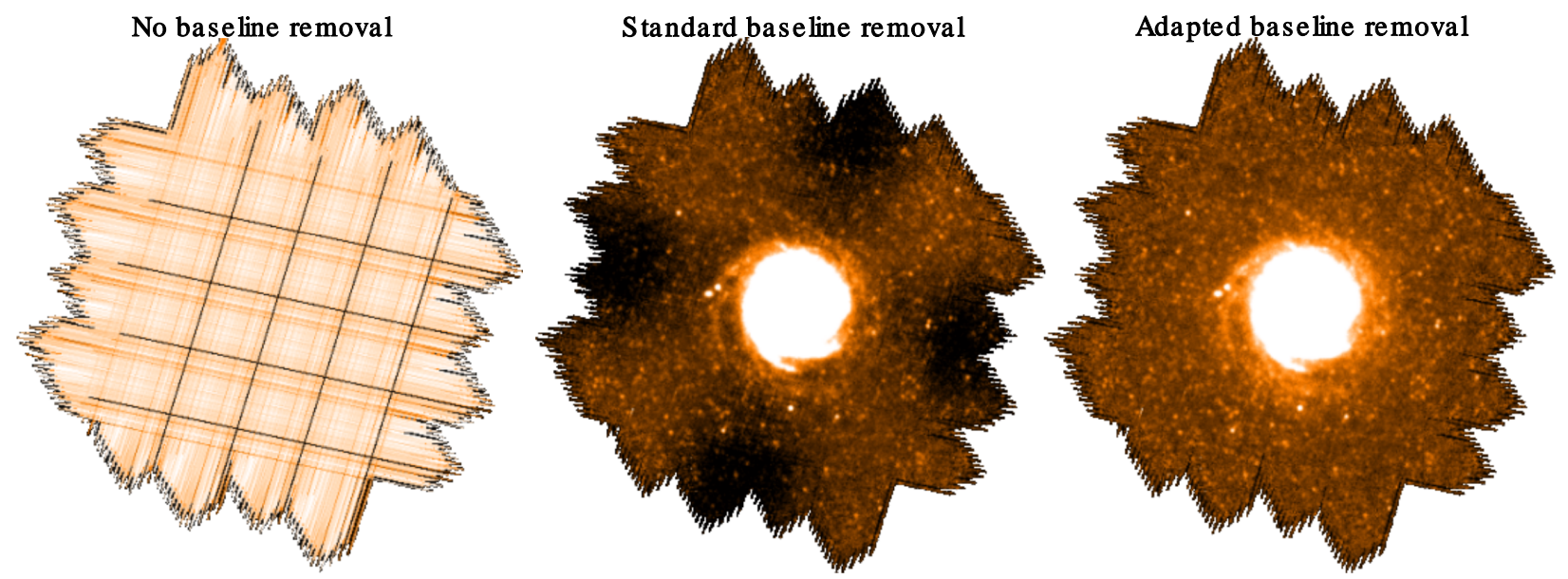

Figure 5. Effect of baseline removal on map of a bright, extended galaxy. The left panel shows the result if the Level 1 data are mapped without any adjustments of the detector DC offsets. The middle panel shows the result of the standard median baseline removal, which leaves visible artifacts since the source biases the median of the bolometer timelines. The right panel shows an adapted baseline removal where only the first and last 100 data samples (away from the galaxy) are used for a robust linear fit of the signal in each scanline.

pointing system. Third, since the telescope pointing and SPIRE detector timelines are sampled at different intervals, the final step is to interpolate the Right Ascension and Declination for each detector sample.

\subsection{Omit Data During Telescope Acceleration}

By default, the scan turnaround data which was used to buffer the scan timelines (Section 3.2) is cut from further analysis. It is possible to override this feature, but the properties of the resultant map edges have not yet been studied carefully. At the conclusion of this pipeline module, the data have reached the 'Level 1' stage.

A minor bug in the Level 0 processing which is not yet understood leads to the scanline boundaries being misplaced by $\sim 1$ second. Effects of this are a slight acceleration at the beginning of nominal scanlines and wavier edges to the maps (visible in Figure 5).

\subsection{Remove Detector Baselines}

In the ideal case of perfect calibration, the Level 1 data could be fed directly into the mapper. However, in reality we need to add another step to adjust the relative DC offsets among the different bolometers (Figure 5). The initial versions of the pipeline (through version 4) do baseline subtraction of each bolometer independently: the median of the timeline is subtracted from each sample. This is a suitable approach for sparse, weak sources, but leaves negative shadows around brighter sources (Figure 5). More sophisticated and interactive solutions such as excluding the region with bright emission from the samples over which the median is calculated - can give better results. Baseline subtraction is discussed further in Section 7.

By using a higher order fit to the timeline, e.g., a linear fit to the samples from the start and end of each scanline, the baseline removal can in addition remove any residual non-astronomical drift left over from the temperature drift correction.

\subsection{Map Making}

In HIPE, two map makers are available. The current default is a naïve mapper which bins each detector sample onto the nearest sky map pixel in a grid centred on the up-linked source RA/Dec.

In the naïve mapper, all of the detector samples falling in one pixel are averaged, and the number of samples is added to the equivalent pixel in the associated coverage map (hit map). In addition, an error map giving the 

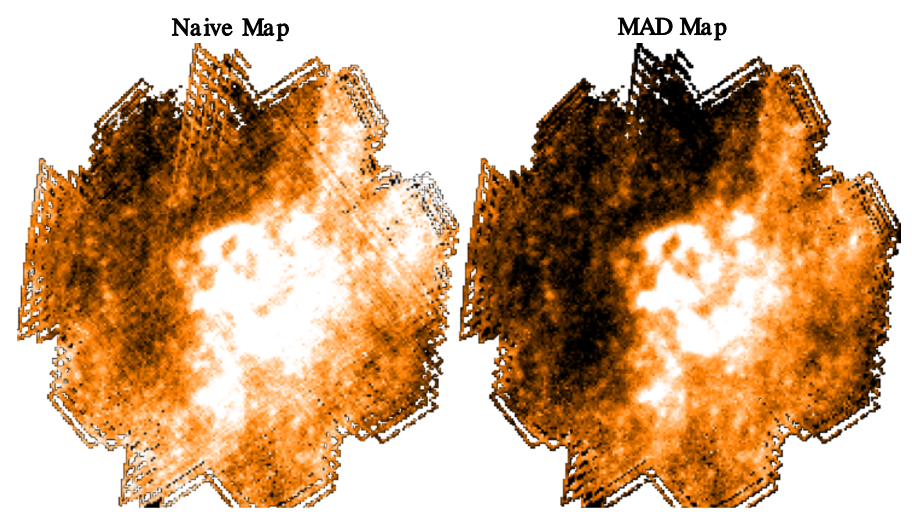

Figure 6. Example of madMap successfully removing the low-level striping visible in the naïve map.

standard deviation of the mean $\sigma_{\mu}$ is calculated as follows:

$$
\sigma_{\mu}=\sqrt{\frac{N \sum_{i} x_{i}^{2}-\left(\sum_{i} x_{i}\right)^{2}}{N^{3}-N^{2}}},
$$

where $x_{i}$ is a detector sample and the sums and total number of samples $N$ apply only to those which are not excluded for poor quality. Currently, we exclude 15 bolometers (dead, excessively noisy, or anomalously slow response) from the analysis.

In addition to the naïve mapper used in the standard pipeline there is a maximum-likelihood mapper (madMap $\left.{ }^{11}\right)$ available. This mapper can be used to remove low-frequency drift (" $1 / \mathrm{f}$ ") noise from bolometer data while preserving the sky signal on large spatial scales. MadMap requires an additional detector-detector noise correlation matrix, which is available in the pipeline version 4. MadMap is still under test for SPIRE scan maps; out-of-the-box, it gives improved maps in some cases (Figure 6) and worse in others.

The current default pixel size is 6/10/14"/pixel for PSW/PMW/PLW respectively. Depending on the mode (SPIRE-only or Parallel) and the number of repetitions, finer pixel sizes of size, e.g., FWHM/4 (4.5", 6.25", and $\left.9^{\prime \prime}\right)$ can be used without introducing many holes with no coverage in the maps.

\section{POINT SOURCE PHOTOMETRY PIPELINE}

The SPIRE Point Source Photometry mode is used for measurement of point source flux densities in a minimum required observing time. The telescope points at a target, and the sky image is both chopped rapidly (at $1 \mathrm{~Hz}$ ) along the spacecraft $Y$ axis and jiggled slowly (typically once every eight chop cycles) in the $Y$ - $Z$ plane with respect to the detector arrays using the instrument Beam Steering Mirror (BSM), in a hexagonal 7-point pattern ( 6 corners and a central position separated by $6^{\prime \prime}$ ). The sky background is removed by chopping $\pm 63^{\prime \prime}$ with the BSM, and any remaining asymmetries in the optics and thermal radiation field of the telescope are removed by nodding with the telescope $126^{\prime \prime}$. The source flux is thus extracted from the difference signal produced by this chopping and nodding action as illustrated in Figure 7.

Each SPIRE Point Source AOT is assembled from building blocks corresponding to a jiggle operation at a single nod position ( $A$ or $B$, see Figure 7 ). A single Jiggle operation building block consists of 8 contiguous on-source, off-source chop cycles at each of 7 jiggle positions (plus an 8th at the original starting jiggle position). This operation is carried out at nod position $A$, then position $B$, repeated at nod position $B$ then finally again at nod position $A$. Thus a single SPIRE Point Source AOT consists of one $A B B A$ nod cycle (the most efficient nodding sequence).

The SPIRE point source mode pipeline, as originally conceived, was described previously. ${ }^{3}$ In Figure 8 we present the current state of the processing pipeline within HIPE version 4. The pipeline processes data from the basic Level 0.5 voltage detector data through to the final flux calibrated, science grade Level 1 and Level 2 data products. 

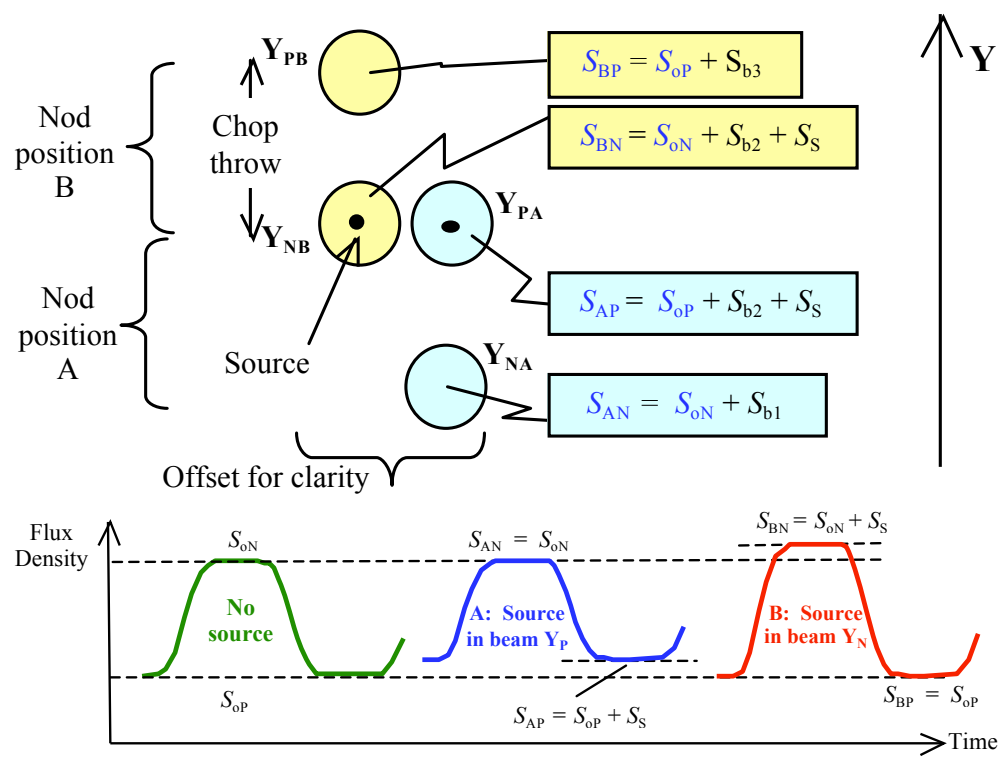

Figure 7. Operation of the SPIRE Point Source Mode. top: Three positions viewed by one detector during the sequence are illustrated. Chopping and nodding are along the spacecraft $Y$ (chop) axis. The two chop positions are denoted $Y_{P}$ and $Y_{N}$, with $Y_{P}$ (positive) being the one with the more positive $Y$ position and $Y_{N}$ being the one with the more negative $Y$ position. The two nod positions are designated $A$ and $B$, with position $B$ being the more positive in $Y$. In the case illustrated, it is assumed that there is a source of flux density $S_{S}$ in the position that is common to both nod positions, and that the sky background varies with position, having values $S_{b 1}, S_{b 2}$, and $S_{b 3}$ in the three positions observed by the detector. $S_{o P}$ and $S_{o N}$ are the flux densities that would be derived from the bolometer outputs in the $Y_{P}$ and $Y_{N}$ beams for completely blank sky. (These are generated entirely locally, and have nothing to do with the sky brightness. $S_{o P}$ and $S_{o N}$ are unequal because the detector does not view the local (instrument and telescope) background identically in the two BSM positions). bottom: Flux density levels measured during chopping and nodding. The lower panel shows example timelines for nod positions $A$ and $B$ (with the source in the right beam for nod position $A$ ), where for simplicity the sky background is taken to be zero.

\subsection{Detector Timeline Processing}

The Level 0 to Level 0.5 processing follows a common pipeline for all observing modes and is descibed earlier in Section 3. The Level 0.5 Point Source mode detector timelines contain signal and mask data as a function of time for a single AOT building block. These building blocks are processed in turn through the pipeline until the De-Nodding stage.

\subsection{BSM Timeline Processing}

In addition to the detector timeline data, the BSM timeline is a Level 0.5 data product containing the motion of the BSM measured as sensor signals in raw ADU. The Compute BSM Angles module converts these raw sensor signals into arcseconds as measured in the spacecraft $Y, Z$ plane by means of a calibration look-up table and feeds this positional information into the SPIRE Pointing Product.

The AOT chop-jiggle pattern is stored in the BSM Operations calibration file as a set of expected sensor positions. The Extract Chop 83 Jiggle Positions module uses this calibration file and the BSM timeline to produce a timeline of chop and jiggle markers used for demodulation.

\subsection{Correction for Instrumental Effects}

The modules that correct for instrumental effects (Electrical Crosstalk, First Level Deglitching, and Flux Conversion) follow the same procedure as the Scan Map Mode described in Section 3, although the optimal parameters for the deglitching process differ slightly from the Scan Map Mode. 


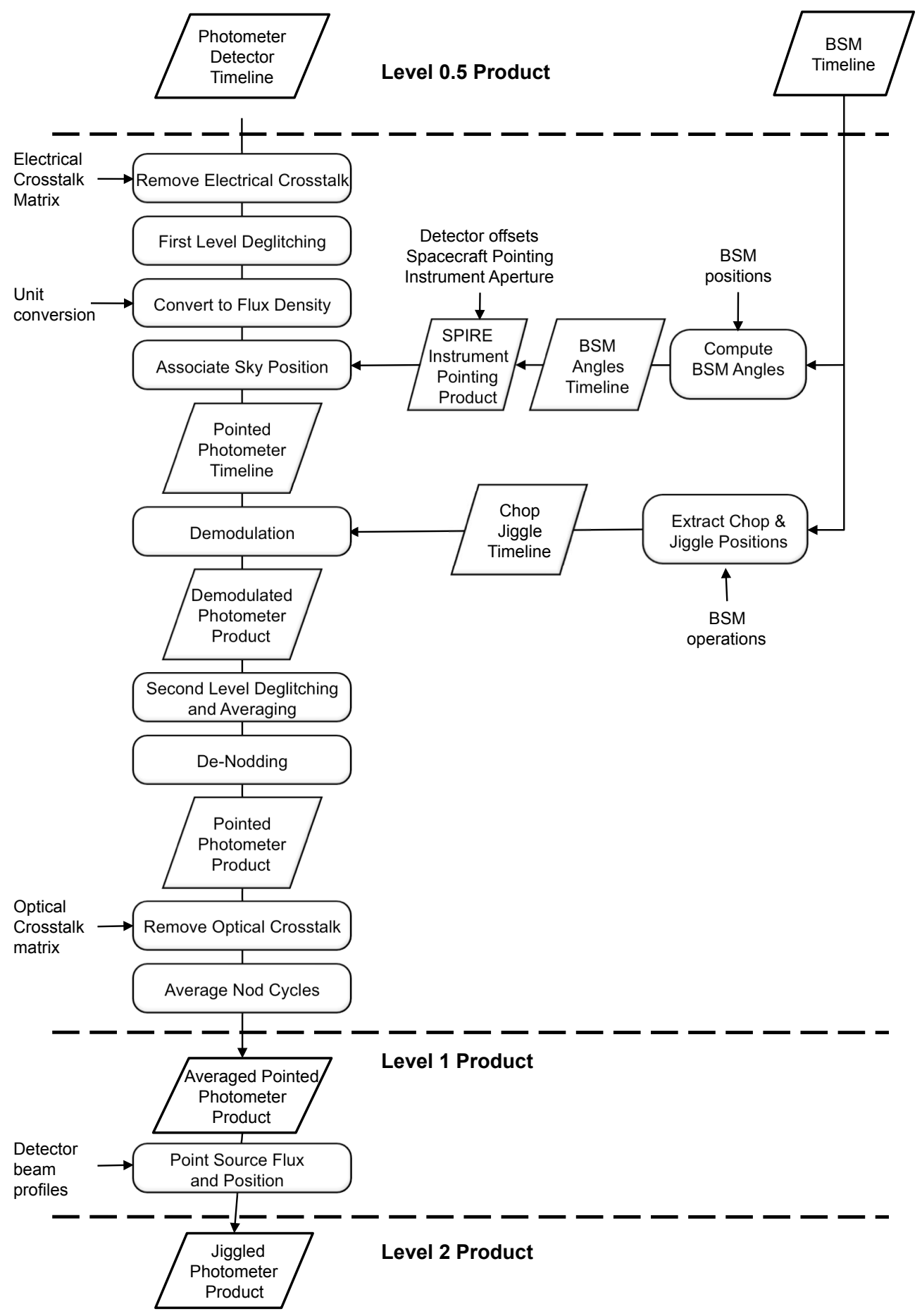

Figure 8. The pipeline flowchart for the SPIRE Point Source Mode showing the processing steps from the Level 0.5 data through to the final Level 1 and Level 2 data products. Processing tasks are shown as rounded rectangles, data products as parallelograms, and calibration files as plain text. 


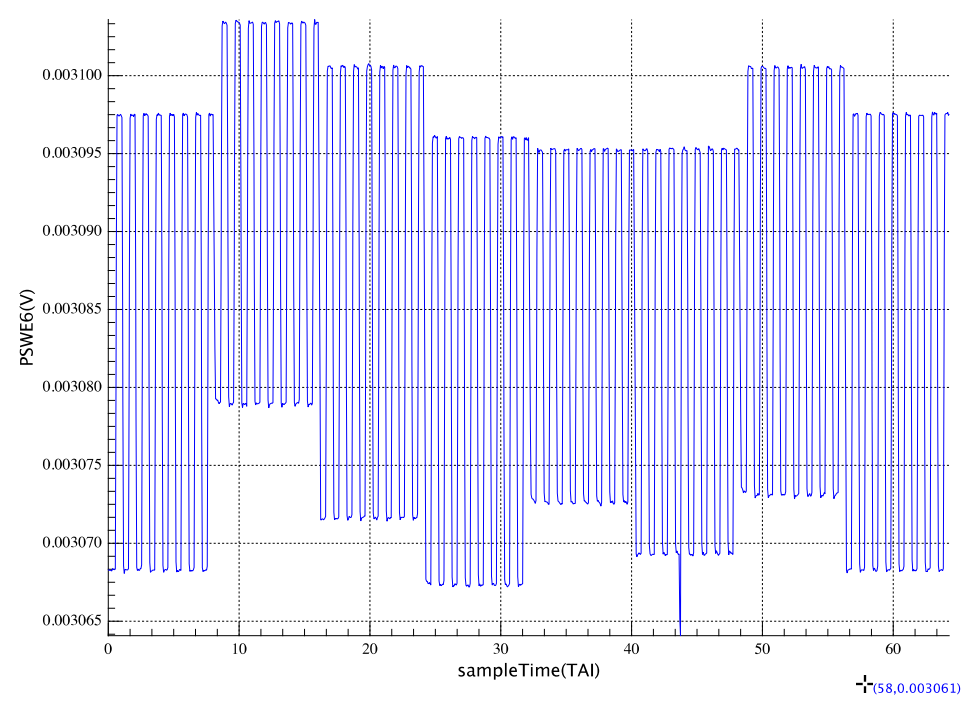

Figure 9. Modulated detector timeline clearly showing the on and off source chopped signal and the 8 Jiggle positions.

\subsection{Merge Detector Samples with Telescope Pointing}

The Associate Sky Position module takes the calibrated detector signal timelines and merges them with the telecope pointing timeline to create Pointed Photometer Timelines. The procedure is identical to that described for the Scan Map pipeline.

\subsection{Demodulation}

The Demodulation module produces a difference signal from data taken at the two chop positions $Y_{P}$ and $Y_{N}$ (See Figure 9). A single building block consists of $N_{\text {chop }}=8$ chop cycles carried out at each of $N_{\text {jigg }}$ Jiggle positions, for all Jiggle positions for all detectors for a single nod position. Within each chop cycle the BSM is sampled 8 times, 4 times in the $Y_{P}$ chop position and 4 times in the $Y_{N}$ chop position (see Figure 7 ). The first sample point in each chop position is deemed to be unstable and is not used. The other 3 samples in the $Y_{P}$ chop position are averaged before demodulation as are the equivalent 3 samples in the $Y_{N}$ chop position. Considering the building blocks at nod position $A$, the demodulated signal for a single chop cycle will be $S_{A}=\left(S_{o P}+S_{b 2}+S_{S}\right)-\left(S_{o N}+S_{b 1}\right)$. At nod position $B$ the demodulated signal will be $S_{A}=\left(S_{o P}+S_{b 3}\right)-\left(S_{o N}+S_{b 2}+S_{S}\right)$. Chop and Jiggle positions are defined by the Chop-Jiggle Timeline Product which contains the chop markers $\left(Y_{P}\right.$ or $\left.Y_{N}\right)$ and the Jiggle Position $\left(N_{\text {Jigg }}\right)$ as a function of time. The resulting Demodulated Photometer Product contains the demodulated signal for each of the $N_{\text {chop }}$ cycles at each jiggle position for each detector.

\subsection{Second-Level Deglitching and Averaging}

For each individual nod position, the $N_{c h o p}$ estimates of the demodulated flux densities can now be deglitched by rejecting outliers (currently set to $5 \sigma$ ) and averaging the remaining samples, to produce mean values and an associated uncertainty at a particular nod position.

\subsection{De-Nodding}

The De-Nod module subtracts out contributions from any asymmetries in the optics and thermal radiation field of the telescope by simply taking the difference between the flux densities in the two nod positions ( $A$ and $B$ for both parts of a typical $A B B A$ repetition). The output from this processing module is a Pointed Photometer Product containing the de-nodded signal for each detector.

\subsection{Remove Optical Crosstalk}

This module follows the same procedure as the Scan Map pipeline described in Section 3. 


\subsection{Average Over Nod Cycles and the Level 1 Product}

If there are more than a single $A B B A$ nod cycle, the Average Nod Cycles module calculates a weighted mean and uncertainty from the separate estimates for each nod cycle. The Level 1 data product for the SPIRE Point Source pipeline is termed an Averaged Pointed Photometer Product (APPP) and contains the demodulated, de-nodded signal at each of the 7 jiggle positions for every detector.

\subsection{Fit Point Source Flux and Position and the Level 2 Product}

For the seven-point jiggle there are eight data points for each detector (for each input APPP), one for each specific jiggle position. This distribution is fitted with a Gaussian approximation to the main beam profile to derive the source flux density and offset with respect to the pointed position. The estimation of flux density and position are carried out independently. In addition, in the case of point source observations, three detectors in each array see the source at some time during the observation: a primary, upper and lower detector set (See Figure 7, currently set to be PSW E6 (prime), E2, E10, PMW D8 (prime), D11, D5, PLW C4 (prime), C2, C6). The primary detector sees the source in both nod cycles; however, the other two detector sets only see the source in one of the nod positions. Thus, in the course of the observation, five different sky positions are viewed by the three detectors (per array). Therefore it is expected that there will be a positive signal in the prime detector and negative signals with half the amplitude in two others. The final Level 2 data product is the Jiggled Photometer Product which contains a single measurement of the flux and position of the target for each of the three detector arrays taking into account all available data.

\section{MAP CALIBRATION CONVENTIONS}

The calibration convention for SPIRE Scan Maps and Jiggled Photometer Products is that the output flux densities are given in Jy per beam. This is discussed in some detail in the SPIRE Observers' Manual, but we touch on some of the key issues here.

Calibration in Jy/beam may be best explained by an example. Consider a 1 Jy point-like source (i.e., of size much smaller than the Herschel diffraction beam) at $\lambda=350 \mu \mathrm{m}$, and ignore random detector noise and inevitable calibration error. Except for a caveat regarding spectral slope (explained below), this source should produce exactly a 1 Jy deflection in the Level 1 product for a PMW detector which is aligned perfectly with the point source. Except for a second caveat regarding map pixel size (also explained below), this same source should also produce a 1 Jy peak signal in the Level 2 map in the case that the source position corresponds exactly with the center of a map pixel.

Now, the caveat about map pixel size: The standard map maker bins the measurements, which have quasicontinuous positional information, into discrete, relatively coarse map pixels. A map pixel with center corresponding exactly to the point source position will collect some measurements with the reported flux density equal to the true flux density. However, the pixel will also accumulate measurements where the source and measurement position are misaligned by up to 0.7 times the pixel width, and the reported flux density for those measurements will be somewhat lower than the true flux density. This average reduction of the map signal is calculated to be $5 \%$ to $7 \%$ for the default pixel scales.

A point source which does not align with the center of a map pixel will experience an additional reduction in the peak observed flux in the map. In this more general case, the true flux can be derived by fitting with the measured beam shape. This is currently being studied by the ICC (with the preliminary result that photometry with $<1 \%$ precision is challenging), and a recommended method will be documented at a later date.

Regarding the caveat about spectral slope: The convention for SPIRE is that true flux densities at 250, 350 , or $500 \mu \mathrm{m}$ are only equal to flux densities reported at the Level 2 stage when the source spectrum $S_{\nu}$ is proportional to $\nu^{-1}$. Furthermore, the convention is to quote all flux densities at $\lambda$ exactly equal to 250, 350, and $500 \mu \mathrm{m}$ and to make color corrections for spectral slope, rather than to compute effective wavelengths. The color corrections have been calculated using the measured Relative Spectral Response Functions (RSRFs), and are a few $\%$ for typical source spectra. 
A resolved source with integrated flux equal to 1 Jy will, of course, produce a signal in the Level 1 and Level 2 products which is less than $1 \mathrm{Jy} /$ beam. Flux densities of extended sources can be measured by summing map pixels and dividing by the beam area:

$$
S=\frac{L^{2} \sum_{i} M_{i}}{\Omega_{\text {beam }}}
$$

where $S$ is the integrated flux, $L^{2}$ is the area of a map pixel, $M$ is the signal in a map pixel, and $\Omega_{\text {beam }}$ is the beam area (tabulated in the SPIRE Observers' Manual).

\section{DATA HANDLING AND ARCHIVING}

By design Herschel operates autonomously. The recorded data from Herschel observations are stored on-board and transmitted to the ground station (either New Norcia, Australia or Cebreros, Spain) during the $\sim 3$ hour of daily telecommunication periods.

The raw satellite, science, and housekeeping telemetry, received by the ground stations, is consolidated in the Mission Operations Centre (MOC) in the European Space Operations Centre (ESOC) in Darmstadt, Germany. The fully consolidated data for a given observational day are then transmitted to the Herschel Science Centre (HSC) at the European Space Astronomy Centre (ESAC) near Madrid, Spain. The raw telemetry is ingested in a special object-oriented database and a flag, indicating that the full consolidated telemetry packets and the necessary auxiliary products (like the satellite attitude history file) are received, triggers automatic data processing using the so-called Systematic Product Generation (SPG) with instrument pipelines developed in the Herschel Common Software System (HCSS).

The SPG pipelines process the data from each astronomical observing request to products at different levels, depending on the observing mode. Upon successful termination, the data are stored in the Herschel Science Archive (HSA). A quality assessment is performed by the HSC, and the principal investigator of the observing program is notified that his/her data are available for retrieval.

The HSA can be accessed via a standard web interface, common to most of the ESA missions (e.g., ISO, Integral, XMM), as well as through HIPE. In general, the observations have a proprietary period of one year. There are some public data already available now: some Science Demonstration data as well as calibration observations performed with standard observing modes. Loading the data in HIPE allows for interactive processing of the observations with the standard or with a modified version of the pipeline.

\section{FUTURE DIRECTIONS}

Within HIPE version 5, there will be a more versatile baseline removal tool available for users to run interactively. This tool allows a robust polynomial fit of a specified order with rejection of outliers. The user can select if the fit should be done on a scan-by-scan basis (memory efficient) or over the entire observation. The purpose of this new tool is to better estimate detector baselines in the presence of sources (Section 3.12).

First tests have shown that a higher order polynomial fit to the baseline of the whole observation is necessary in many cases to successfully run madMap. The aforementioned task will provide this functionality and allow the subsequent final validation of madMap.

An alternative approach to detector baseline subtraction is to use the cross-linked scans to determine the relative detector offsets at regions of overlap. This process implicitly uses the map of the sky and is easiest to implement with an iterative approach. ${ }^{12,13}$ The least-squares version of this iterative destriper minimizes:

$$
\chi^{2}=\sum_{b, i}\left(\frac{d_{b, i}-C_{b}-g_{b} M\left(\mathbf{r}_{b, i}\right)}{\sigma_{b, i}}\right)^{2},
$$

where $b$ is the bolometer index, $i$ is the time index, $d$ is the Level 1 detector sample, $C$ is the detector offset, $M$ is the flux density at position $\mathbf{r}$ in the map, and $g_{b}$ is an optional correction to the bolometer gain. $C$ could be determined on an observation-by-observation or scanline-by-scanline basis. An iterative destriper is under evaluation and is planned for HIPE version 5 . 
Iterative mapping also facilitates second-level deglitching - rejection of outliers based on multiple measurements of the same map pixel - but this is not necessarily tied to the iterative destriper. The ICC will consider the option of adding second-level deglitching in the processing between Levels 1 and 2 for later versions of HIPE.

We conclude by noting that the SPIRE photometer pipelines through HIPE version 3 that were used for Science Demonstration Phase have already produced high quality data. Calibration has moved part way from pre-flight estimates to in-flight measurements; with the imminent release of nonlinearity correction and absolute flux calibration based on flight data and Neptune observations in HIPE version 4, we can expect further gains in accuracy. Various other pipeline enhancements are anticipated in the next six to twelve months, allowing SPIRE users to extract the full capabilities of the instrument in their science data analyses.

\section{ACKNOWLEDGMENTS}

SPIRE has been developed by a consortium of institutes led by Cardiff Univ. (UK) and including Univ. Lethbridge (Canada); NAOC (China); CEA, LAM (France); IFSI, Univ. Padua (Italy); IAC (Spain); Stockholm Observatory (Sweden); Imperial College London, RAL, UCL-MSSL, UKATC, Univ. Sussex (UK); Caltech, JPL, NHSC, Univ. Colorado (USA). This development has been supported by national funding agencies: CSA (Canada); NAOC (China); CEA, CNES, CNRS (France); ASI (Italy); MCINN (Spain); SNSB (Sweden); STFC (UK); and NASA (USA). HCSS, HSpot, and HIPE are a joint development by the Herschel Science Ground Segment Consortium, consisting of ESA, the NASA Herschel Science Center, and the HIFI, PACS and SPIRE consortia.

We thank the SPIRE SAG2 for the permission to show their map of NGC 6822 and our commissioning phase map of one of their objects (M83).

\section{REFERENCES}

[1] Pilbratt, G. L., Riedinger, J. R., Passvogel, T., Crone, G., Doyle, D., Gageur, U., Heras, A. M., Jewell, C., Metcalfe, L., Ott, S., and Schmidt, M., "Herschel Space Observatory - An ESA facility for far-infrared and submillimetre astronomy," ArXiv e-prints (May 2010).

[2] Griffin, M. J., Abergel, A., Abreu, A., Ade, P. A. R., André, P., Augueres, J., Babbedge, T., Bae, Y., Baillie, T., Baluteau, J., Barlow, M. J., Bendo, G., Benielli, D., Bock, J. J., Bonhomme, P., Brisbin, D., Brockley-Blatt, C., Caldwell, M., Cara, C., Castro-Rodriguez, N., Cerulli, R., Chanial, P., Chen, S., Clark, E., Clements, D. L., Clerc, L., Coker, J., Communal, D., Conversi, L., Cox, P., Crumb, D., Cunningham, C., Daly, F., Davis, G. R., De Antoni, P., Delderfield, J., Devin, N., Di Giorgio, A., Didschuns, I., Dohlen, K., Donati, M., Dowell, A., Dowell, C. D., Duband, L., Dumaye, L., Emery, R. J., Ferlet, M., Ferrand, D., Fontignie, J., Fox, M., Franceschini, A., Frerking, M., Fulton, T., Garcia, J., Gastaud, R., Gear, W. K., Glenn, J., Goizel, A., Griffin, D. K., Grundy, T., Guest, S., Guillemet, L., Hargrave, P. C., Harwit, M., Hastings, P., Hatziminaoglou, E., Herman, M., Hinde, B., Hristov, V., Huang, M., Imhof, P., Isaak, K. J., Israelsson, U., Ivison, R. J., Jennings, D., Kiernan, B., King, K. J., Lange, A. E., Latter, W., Laurent, G., Laurent, P., Leeks, S. J., Lellouch, E., Levenson, L., Li, B., Li, J., Lilienthal, J., Lim, T., Liu, J., Lu, N., Madden, S., Mainetti, G., Marliani, P., McKay, D., Mercier, K., Molinari, S., Morris, H., Moseley, H., Mulder, J., Mur, M., Naylor, D. A., Nguyen, H., O'Halloran, B., Oliver, S., Olofsson, G., Olofsson, H., Orfei, R., Page, M. J., Pain, I., Panuzzo, P., Papageorgiou, A., Parks, G., Parr-Burman, P., Pearce, A., Pearson, C., Pérez-Fournon, I., Pinsard, F., Pisano, G., Podosek, J., Pohlen, M., Polehampton, E. T., Pouliquen, D., Rigopoulou, D., Rizzo, D., Roseboom, I. G., Roussel, H., Rowan-Robinson, M., Rownd, B., Saraceno, P., Sauvage, M., Savage, R., Savini, G., Sawyer, E., Scharmberg, C., Schmitt, D., Schneider, N., Schulz, B., Schwartz, A., Shafer, R., Shupe, D. L., Sibthorpe, B., Sidher, S., Smith, A., Smith, A. J., Smith, D., Spencer, L., Stobie, B., Sudiwala, R., Sukhatme, K., Surace, C., Stevens, J. A., Swinyard, B. M., Trichas, M., Tourette, T., Triou, H., Tseng, S., Tucker, C., Turner, A., Vaccari, M., Valtchanov, I., Vigroux, L., Virique, E., Voellmer, G., Walker, H., Ward, R., Waskett, T., Weilert, M., Wesson, R., White, G. J., Whitehouse, N., Wilson, C. D., Winter, B., Woodcraft, A. L., Wright, G. S., Xu, C. K., Zavagno, A., Zemcov, M., Zhang, L., and Zonca, E., "The Herschel-SPIRE instrument and its in-flight performance," ArXiv e-prints (May 2010). 
[3] Griffin, M., Dowell, C. D., Lim, T., Bendo, G., Bock, J., Cara, C., Castro-Rodriguez, N., Chanial, P., Clements, D., Gastaud, R., Guest, S., Glenn, J., Hristov, V., King, K., Laurent, G., Lu, N., Mainetti, G., Morris, H., Nguyen, H., Panuzzo, P., Pearson, C., Pinsard, F., Pohlen, M., Polehampton, E., Rizzo, D., Schulz, B., Schwartz, A., Sibthorpe, B., Swinyard, B., Xu, K., and Zhang, L., "The Herschel-SPIRE photometer data processing pipeline," in [Space Telescopes and Instrumentation 2008: Optical, Infrared, and Millimeter], Proc. SPIE $\mathbf{7 0 1 0}$ (Aug. 2008).

[4] Ott, S. in [Astronomical Data Analysis Software and Systems XIX], Mizumoto, Y., Morita, K.-I., and Ohishi, M., eds., ASP Conference Series (2010).

[5] Kilbourne, C. A., Boyce, K. R., Brown, G. V., Cottam, J., Figueroa-Feliciano, E., Fujimoto, R., Furusho, T., Ishisaki, Y., Kelley, R. L., McCammon, D., Mitsuda, K., Morita, U., Porter, F. S., Ota, N., Saab, T., Takei, Y., and Yamamoto, M., "Analysis of the Suzaku/XRS background," Nuclear Instruments and Methods in Physics Research A 559, 620-622 (Apr. 2006).

[6] Ordénovic, C., Surace, C., Torrésani, B., Llebaria, A., and Baluteau, J. P., "Use of a local regularity analysis by a wavelet analysis for glitch detection," in [Society of Photo-Optical Instrumentation Engineers (SPIE) Conference Series], A. G. Tescher, ed., Proc. SPIE 5909, 556-567 (Aug. 2005).

[7] Swinyard, B. M., Ade, P., Baluteau, J., Aussel, H., Barlow, M. J., Bendo, G. J., Benielli, D., Bock, J., Brisbin, D., Conley, A., Conversi, L., Dowell, A., Dowell, D., Ferlet, M., Fulton, T., Glenn, J., Glauser, A., Griffin, D., Griffin, M., Guest, S., Imhof, P., Isaak, K., Jones, S., King, K., Leeks, S., Levenson, L., Lim, T. L., Lu, N., Makiwa, G., Naylor, D., Nguyen, H., Oliver, S., Panuzzo, P., Papageorgiou, A., Pearson, C., Pohlen, M., Polehampton, E., Pouliquen, D., Rigopoulou, D., Ronayette, S., Roussel, H., Rykala, A., Savini, G., Schulz, B., Schwartz, A., Shupe, D., Sibthorpe, B., Sidher, S., Smith, A. J., Spencer, L., Trichas, M., Triou, H., Valtchanov, I., Wesson, R., Woodcraft, A., Xu, C. K., Zemcov, M., and Zhang, L., "In-flight calibration of the Herschel-SPIRE instrument," ArXiv e-prints (May 2010).

[8] Moreno, R., [Thèse de Doctorat], Université de Paris VI (1998).

[9] Moreno, R., [Neptune and Uranus planetary brightness temperature tabulation], available frm ESA Herschel Science Center (2010).

[10] Schulz, B., Bock, J. J., Lu, N., Nguyen, H. T., Xu, C. K., Zhang, L., Dowell, C. D., Griffin, M. J., Laurent, G. T., Lim, T. L., and Swinyard, B. M., "Noise performance of the Herschel-SPIRE bolometers during instrument ground tests," in [Society of Photo-Optical Instrumentation Engineers (SPIE) Conference Series], Proc. SPIE $\mathbf{7 0 2 0}$ (Aug. 2008).

[11] Cantalupo, C. M., Borrill, J. D., Jaffe, A. H., Kisner, T. S., and Stompor, R., "MADmap: A Massively Parallel Maximum Likelihood Cosmic Microwave Background Map-maker," Astrophysical Journal Supplement Series 187, 212-227 (Mar. 2010).

[12] Bendo, G. J., Wilson, C. D., Pohlen, M., Sauvage, M., Auld, R., Baes, M., Barlow, M. J., Bock, J. J., Boselli, A., Bradford, M., Buat, V., Castro-Rodriguez, N., Chanial, P., Charlot, S., Ciesla, L., Clements, D. L., Cooray, A., Cormier, D., Cortese, L., Davies, J. I., Dwek, E., Eales, S. A., Elbaz, D., Galametz, M., Galliano, F., Gear, W. K., Glenn, J., Gomez, H. L., Griffin, M., Hony, S., Isaak, K. G., Levenson, L. R., Lu, N., Madden, S., O'Halloran, B., Okumura, K., Oliver, S., Page, M. J., Panuzzo, P., Papageorgiou, A., Parkin, T. J., Pérez-Fournon, I., Rangwala, N., Rigby, E. E., Roussel, H., Rykala, A., Sacchi, N., Schulz, B., Schirm, M. R. P., Smith, M. W. L., Spinoglio, L., Stevens, J. A., Sundar, S., Symeonidis, M., Trichas, M., Vaccari, M., Vigroux, L., Wozniak, H., Wright, G. S., and Zeilinger, W. W., "The Herschel Space Observatory view of dust in M81," ArXiv e-prints (May 2010).

[13] Levenson, L. R. and et al., "HerMES: Science Demonstration Phase Maps," Monthly Noticies of the Royal Astronomical Society, in preparation (2010). 